\title{
A Novel Photonic Oscillator
}

\author{
X. S. Yao and L. Maleki \\ Tracking Systems and Applications Section
}

\begin{abstract}
We report a novel oscillator for photonic RF systems. This oscillator is capable of generating high-frequency signals up to $70 \mathrm{GHz}$ in both electrical and optical domains and is a special voltage-controlled oscillator with an optical output port. It can be used to make a phase-locked loop (PLL) and perform all functions that a PLL is capable of for photonic systems. It can be synchronized to a reference source by means of optical injection locking, electrical injection locking, and PLL. It can also be self-phase locked and self-injection locked to generate a high-stability photonic $R F$ reference. Its applications include high-frequency reference regeneration and distribution, high-gain frequency multiplication, comb-frequency and square-wave generation, carrier recovery, and clock recovery. We anticipate that such photonic voltage-controlled oscillators (VCOs) will be as important to photonic RF systems as electrical VCOs are to electrical RF systems.
\end{abstract}

\section{Introduction}

RF oscillators, especially voltage controlled oscillators (VCOs), are essential to RF communication, broadcasting, and receiving systems. Their functions include generating, tracking, cleaning, amplifying, and distributing RF carriers. The VCOs in a phase-locked loop configuration can also be used for clock recovery, carrier recovery, signal modulation and demodulation, and frequency synthesizing.

Photonic RF systems [1-3] embed photonic technology into the traditional RF systems. In particular, in a photonic RF system, optical waves are used as a carrier to transport RF signals through optical fiber to remote locations. In addition, some of the RF signal-processing functions, such as signal mixing [4], antenna beam steering [5,6], and signal filtering [7,8] can also be accomplished optically. The photonic technology offers the advantages of low loss, light weight, high frequency, high security, remoting capability, and immunity to electromagnetic interference and, therefore, is desirable in many RF systems.

Traditional RF oscillators cannot meet all the requirements of photonic RF systems. Because photonic RF systems involve RF signals in both optical and electrical domains, an ideal oscillator for the photonic systems should be able to generate RF signals in both optical and electrical domains. In addition, it should be possible to synchronize or control the oscillator by both electrical and optical references or signals.

Presently, generating a high-frequency RF signal in the optical domain is usually done by modulating a diode laser or an external electro-optical (E/O) modulator using a high-frequency stable electrical signal from a local oscillator (LO). Such an LO signal is generally obtained by multiplying a low-frequency reference (e.g., a quartz oscillator) to the required high frequency, say $32 \mathrm{GHz}$, with several stages of 
multipliers and amplifiers. Consequently, the resulting system is bulky, complicated, inefficient, and costly. An alternative way to generate photonic RF carriers is to mix two lasers with different optical frequencies [9]. However, the resulting bandwidth of the signal is wide (limited by the spectral width of the lasers, typically greater than tens of kilohertz) and the frequency stability of the beat signal is poor, caused by the drift of the optical frequency of the two lasers.

We report here a novel photonic oscillator [10] that meets the special requirements for the photonic $\mathrm{RF}$ systems. This oscillator is capable of generating stable signals at frequencies up to $70 \mathrm{GHz}$ (limited by the speed of the $\mathrm{E} / \mathrm{O}$ modulator and photoreceiver) in both electrical and optical domains. We show that the photonic oscillator is a special VCO with both optical and electrical outputs. It can be used to make a phase-locked loop (PLL) and perform all functions that a PLL is capable of for photonic systems. It can also be phase locked to a remote reference through optical injection and, thus, is useful for high-frequency reference regeneration and distribution. We have also demonstrated carrier recovery, clock recovery, high-gain frequency multiplication, and comb-frequency and square-wave generation using the photonic oscillator. By using self-phase-locked loop and self-injection-locking techniques we have developed, high-stability photonic milliwave references can be generated with the photonic oscillator. We anticipate that such photonic VCOs will be as important to photonic RF systems as electrical VCOs are to electrical RF systems.

\section{Device Description}

In a photonic oscillator [10], light from one of the output ports of the E/O modulator is detected by the photodetector and is then amplified, filtered, and fed back to the electrical input port of the modulator, as shown in Fig. 1(a). If the modulator is properly biased and the open-loop gain of the feedback loop is properly chosen, self-electro-optic oscillation will be sustained. Because both optical and electrical processes are involved in the oscillation, both the optical subcarrier and the electrical signal will be generated simultaneously. Note that this ring oscillator is inherently unidirectional, immune to the back reflections in the loop, and therefore generically stable.

The photonic oscillator can be represented by a simple functional block diagram, shown in Fig. 1(b). It is a six-port device with both optical and electrical injection ports, both optical and electrical output ports, and two voltage-controlling ports. One of the controlling ports is simply the bias port of the $\mathrm{E} / \mathrm{O}$ modulator, and the other one is connected to a fiber stretcher for controlling the loop length. As will be shown below, the two injection ports can be used to injection lock the photonic oscillator to a reference source either optically or electrically. The two output ports provide outputs with an RF carrier in both optical and electrical forms. Finally, the two controlling ports can be used to tune the oscillation frequency and to make the photonic oscillator a VCO. The six ports collectively make interfacing the oscillator and a photonic RF system much simpler.

We have built several such photonic oscillators using different modulators and have generated optical subcarriers as high as $9.2 \mathrm{GHz}$, using a diode-pumped YAG laser at $1310 \mathrm{~nm}$. Figure 2(a) shows the generated RF signal at $9.2 \mathrm{GHz}$, and Fig. 2(b) shows the generated signal at $100 \mathrm{MHz}$. In both cases, the photonic oscillators were free running and no effort was made to reduce the noise. For comparison, signal from a Hewlett Packard (HP)8656A signal generator is also shown in Fig. 2(b), and the photonic oscillator clearly has higher spectral purity than the HP8656A.

\section{Steady-State Characteristics}

\section{A. Oscillation Threshold}

To start oscillation from noise, the open-loop gain of the photonic oscillator must be larger than 1 . The open-loop gain of the photonic oscillator is simply the RF power gain of an externally modulated photonic link and is given by [11] 
(a) BIAS

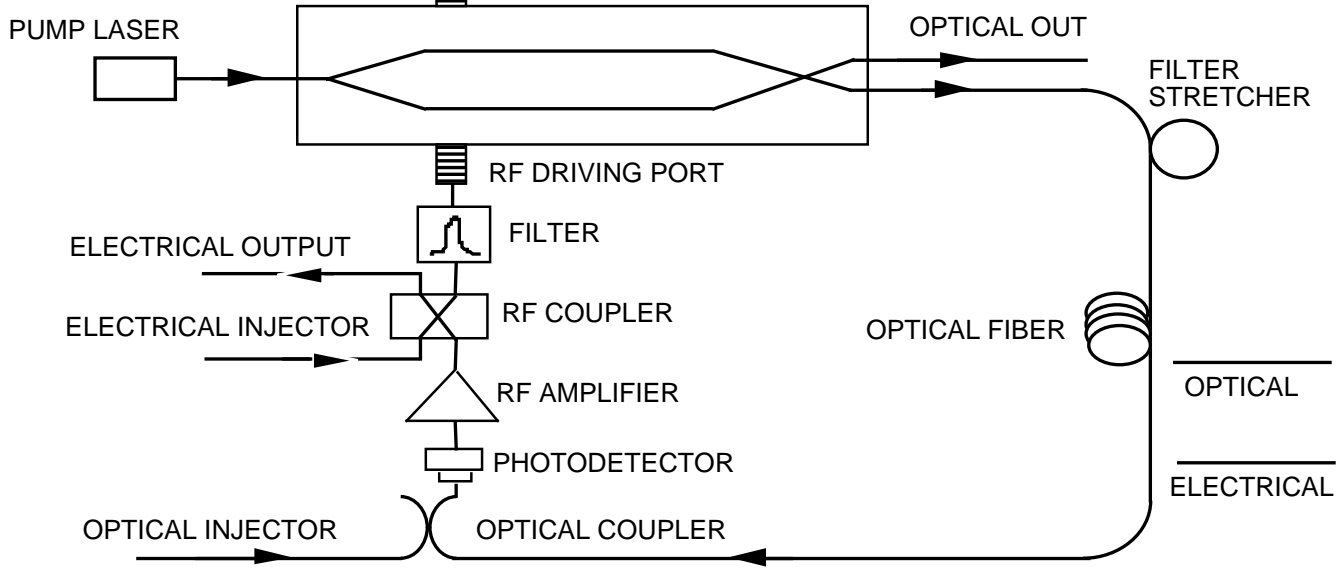

(b)

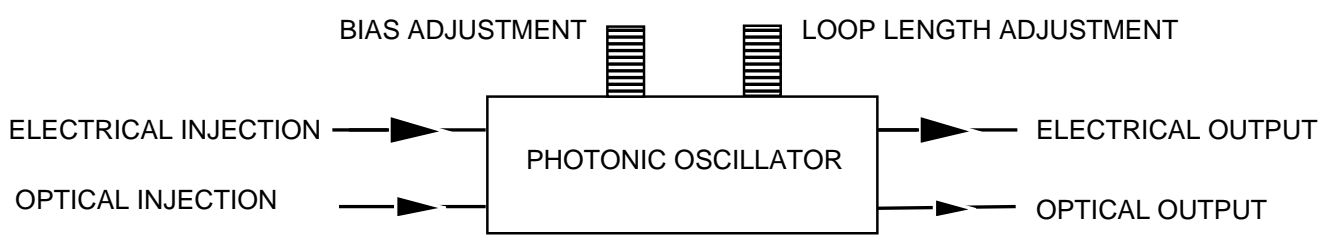

Fig. 1. Device description of the photonic oscillator: (a) device construction and (b) functional diagram.
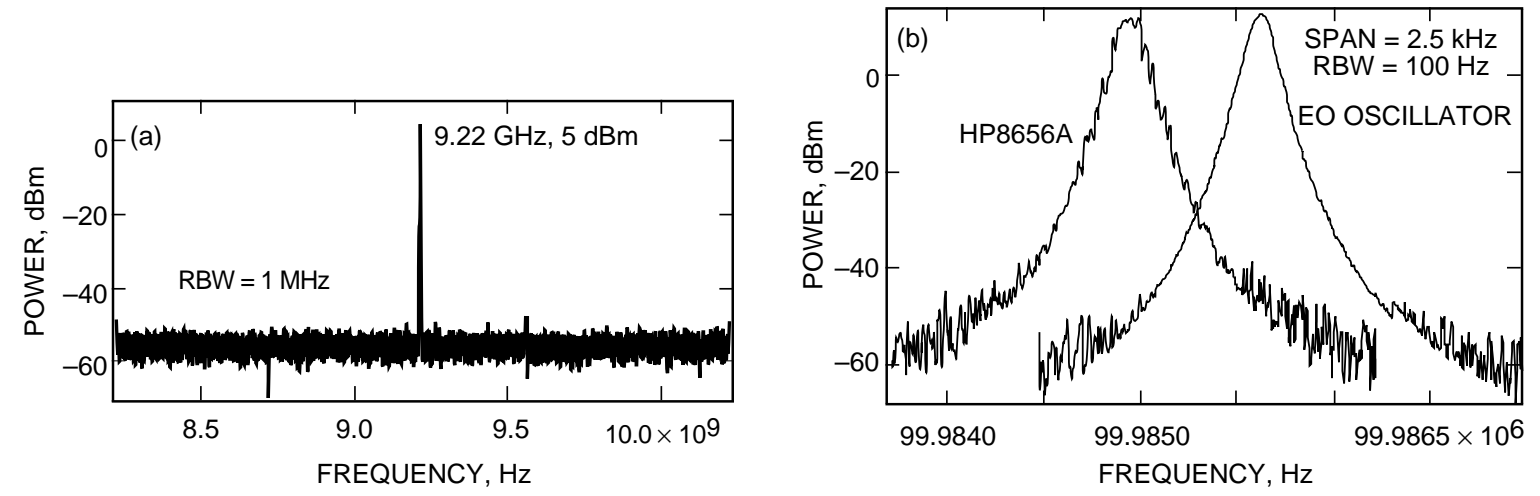

Fig. 2. Spectra of free-running photonic oscillators; oscillation at (a) $9.22 \mathrm{GHz}$ and (b) $100 \mathrm{MHz}$.

$$
G_{\text {open }}^{2}=G_{a m p}^{2} \pi^{2} \frac{I_{p h}^{2} R_{L}}{V_{\pi}^{2} / R_{m}}
$$

where $G_{\text {open }}$ is the open-loop voltage gain, $I_{p h}$ is the photocurrent in the receiver, $R_{L}$ is the load resistance of the receiver, $R_{m}$ is the input impedance of the modulator, $V_{\pi}$ is the half-wave voltage of the modulator, and $G_{a m p}$ is the voltage gain of the amplifier following the photoreceiver. In Eq. (1), the modulator is assumed to be of a Mach-Zehnder type and is biased at quadrature. The oscillation condition of the photonic oscillator is, thus, $G_{\text {open }} \geq 1$. For $R_{L}=R_{m} \equiv R$, this condition becomes 


$$
I_{p h} R G_{a m p} \geq \frac{V_{\pi}}{\pi}
$$

It is important to notice that the amplifier in the loop is not a necessary condition for oscillation. So long as $I_{p h} R \geq V_{\pi} / \pi$ is satisfied, no amplifier is needed $\left(G_{a m p}=1\right)$. It is the optical power from the pump laser that actually supplies the necessary energy for the photonic oscillator. This property is significant because it enables the photonic oscillator to be powered remotely using an optical fiber. In addition, the elimination of the amplifier in the loop also eliminates the amplifier noise, resulting in a more stable oscillator. For a modulator with a $V_{\pi}$ of $3.14 \mathrm{~V}$ and an impedance $R$ of $50 \Omega$, a photocurrent of $20 \mathrm{~mA}$ is required for sustaining the photonic oscillation without an amplifier. This corresponds to an optical power of $25 \mathrm{~mW}$, taking the responsivity $\rho$ of the photodetector to be $0.8 \mathrm{~A} / \mathrm{W}$.

\section{B. Oscillation Amplitude}

The optical power from the output port that forms the loop is related to an applied voltage $V(t)$ by [11]

$$
P(t)=\frac{\alpha P_{o}}{2}\left\{1-\eta \sin \pi \frac{V(t)+V_{B}}{V_{\pi}}\right\}
$$

where $\alpha$ is the fractional insertion loss of the modulator, $V_{B}$ is the bias voltage, and $\eta$ relates to the extinction ratio of the modulator by $(1+\eta) /(1-\eta)$. If $P(t)$ has a positive slope as a function of driving voltage $V(t)$, the modulator is said to be positively biased; otherwise it is negatively biased. Consequently, if the value of $V_{B}$ is chosen such that $V_{B}=0$, the modulator is biased at negative quadrature, while when $V_{B}=V_{\pi}$, the modulator is biased at positive quadrature. Note that in most externally modulated photonic links, the E/O modulators can be biased at either positive or negative quadrature without affecting their performance. However, as will be seen next, the biasing polarity will have an important effect on the operation of the photonic oscillator.

For the photonic oscillator, the applied voltage $V(t)$ is just the photovoltage after the filter in the loop: $V(t)=\rho R G_{a m p} P(t)$, where $\rho$ is the responsivity of the detector, $R$ is the loop impedance, and $G_{a m p}$ is the amplifier voltage gain. The recurrence relation of the oscillating signal in the loop can be easily obtained from Eq. (3):

$$
u(t)=u_{o}\left\{1-\eta \sin \pi\left[u(t-\tau)+u_{B}\right]\right\}
$$

where $\tau$ is the loop delay time and $u_{o}, u_{B}$, and $u(t)$ are the normalized photovoltage, normalized bias voltage, and normalized oscillation voltage, respectively. They are defined as

$$
\begin{aligned}
u_{o} & \equiv \frac{I_{p h} R G_{a m p}}{V_{\pi}} \\
u_{B} & =\frac{V_{B}}{V_{\pi}} \\
u(t) & \equiv \frac{V(t)}{V_{\pi}}
\end{aligned}
$$

In Eq. (5), $I_{p h}=\alpha \rho P_{o} / 2$. Equation (4) simply relates the oscillation voltage at $t$ with that of an earlier time, $t-\tau$. 
At steady state, the oscillating voltage should repeat itself after a round trip in the loop, that is, $u(t)=u(t-\tau)$. This is the self-consistent condition. For the case when only one frequency $\omega_{o}$ is allowed to oscillate by putting a filter in the loop, the solution to Eq. (4) has the form

$$
u(t)=a \sin \left(\omega_{o} t+\phi_{o}\right)
$$

where $a$ is the normalized oscillation amplitude and $\phi_{o}$ is the phase. Note that in writing down Eq. (8) this way, the observation point of the field is chosen to be right after the filter. Substituting Eq. (8) in Eq. (4) and using the self-consistent condition, we obtain

$$
a \sin \left(\omega_{o} t+\phi_{o}\right)=u_{o}\left\{1-\eta \sin \pi\left\{a \sin \left[\omega_{o}(t-\tau)+\phi_{o}\right]+u_{B}\right\}\right\}
$$

Expanding the right-hand side of Eq. (9) using the Bessel function and picking out the term with the fundamental frequency component, we obtain

$$
a \sin \left(\omega_{o} t+\phi_{o}\right)=-2 u_{o} \eta J_{1}(\pi a) \cos \pi u_{B} \sin \left(\omega_{o} t+\phi_{o}-\omega_{o} \tau\right)
$$

To simplify the discussion, we will restrict ourselves to the case when $J_{1}(\pi a) \geq 0$ or $a \equiv V_{\text {osc }} / V_{\pi} \leq 1.21$, since in the majority of cases, the oscillation amplitude $V_{o s c}$ of the oscillator is much less than $V_{\pi}$. With this restriction, we obtain form Eq. (10) the following relations for determining the oscillation amplitude and frequency:

$$
\begin{aligned}
J_{1}(\pi a) & =\frac{\pi a}{2 G_{\text {open }}} \\
f_{o} & =\frac{k+(1 / 2)}{\tau} \text { for } \cos \pi u_{B}>0 \\
f_{o} & =\frac{k}{\tau} \text { for } \cos \pi u_{B}<0
\end{aligned}
$$

where $f_{o}=\omega_{o} / 2 \pi$ and $k$ is an integer. It is interesting to notice from Eq. (12) that the oscillation frequency depends on the biasing polarity of the modulator. For negative biasing $\left(\cos \pi u_{B}>0\right)$, the fundamental frequency is $1 /(2 \tau)$, while for positive biasing $\left(\cos \pi u_{B}<0\right)$, the fundamental frequency is doubled to $1 / \tau$.

In Eq. (11), $G_{\text {open }}$ is the open-loop voltage gain of the oscillator and is defined as

$$
G_{o p e n} \equiv \frac{\pi I_{p h} R G_{a m p} \eta\left|\cos \pi u_{B}\right|}{V_{\pi}}
$$

For the ideal case when $\eta=1$ and $\left|\cos \pi u_{B}\right|=1$, it is the same as that given in Eq. (1). The amplitude of the oscillation can be obtained by solving Eq. (11) graphically, and the result is shown in Fig. 3(a).

Equation (9) can also be solved by expanding its right-hand side into a Taylor series, and the resulting normalized oscillation amplitude is 


$$
\begin{array}{ll}
a \equiv \frac{V_{\text {osc }}}{V_{\pi}}=\frac{2 \sqrt{2}}{\pi} \sqrt{1-\frac{1}{G_{\text {open }}}} & \text { third order expansion } \\
a \equiv \frac{V_{\text {osc }}}{V_{\pi}}=\frac{2 \sqrt{3}}{\pi}\left(1-\frac{1}{\sqrt{3}} \sqrt{\frac{4}{G_{\text {open }}}-1}\right)^{1 / 2} \quad \text { fifth order expansion }
\end{array}
$$

From Eq. (14), one can easily see that the threshold condition for the oscillation is $G_{\text {open }} \geq 1$ or $I_{p h} R G_{a m p} \eta\left|\cos u_{B}\right| \geq V_{\pi} / \pi$. The oscillation frequency obtained using this procedure is the same as Eq. (12).

Figure 3(a) shows the normalized oscillation amplitude as a function of $G_{\text {open }}$ obtained from Eq. (11), Eq. (14a) and Eq. (14b), respectively. Comparing the three theoretical curves, one can see that, for $G_{\text {open }} \leq 1.57$, the third-order expansion result is a good approximation. For $G_{\text {open }} \leq 3.14$, the fifth-order expansion result is a good approximation. Figure 3(b) shows the experimental data, and they are in good agreement with the theoretical results of Eq. (14a).
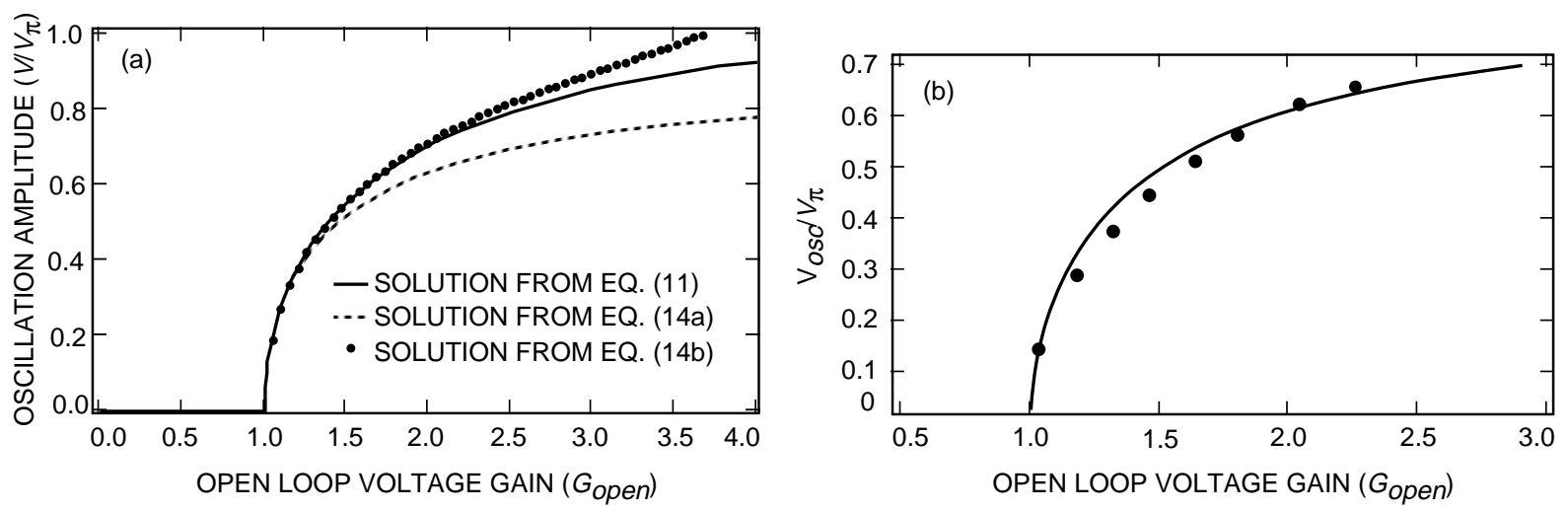

Fig. 3. Oscillation amplitude as a function of photovoltage: (a) theoretical curves and (b) experimental data.

\section{Voltage-Controlled Oscillator Functions}

As mentioned above, the oscillation frequency of the photonic oscillator can be tuned by changing the loop length using a piezoelectric stretcher. The frequency change $\Delta f$ is given by $\Delta f=-f_{o} \Delta L / L$, where $L$ is the loop length, $\Delta L$ is the loop-length change, and $f_{o}$ is the nominal oscillation frequency. However, the tuning sensitivity $(\mathrm{Hz} / \mathrm{V})$ is expected to be small.

The oscillation frequency can also be tuned by changing the bias voltage of the E/O modulator. Figure 4(a) shows that the frequency detuning of the oscillator is linearly proportional to the bias voltage, with a slope of $38.8 \mathrm{kHz} / \mathrm{V}$. The output power of the oscillator remains relatively unchanged in a wide voltage range, as shown in Fig. 4(b). This result is significant because it provided a simple way to tune the oscillation frequency with high sensitivity and is instrumental in realizing PLL using the novel photonic oscillator, as will be discussed next.

\section{Synchronization and Stabilization}

\section{A. Injection Locking}

Injection locking $[12,13]$ is a commonly used technique for synchronizing an oscillator with a reference frequency. A unique aspect of the photonic oscillator is that it can be injection locked by either an optical 

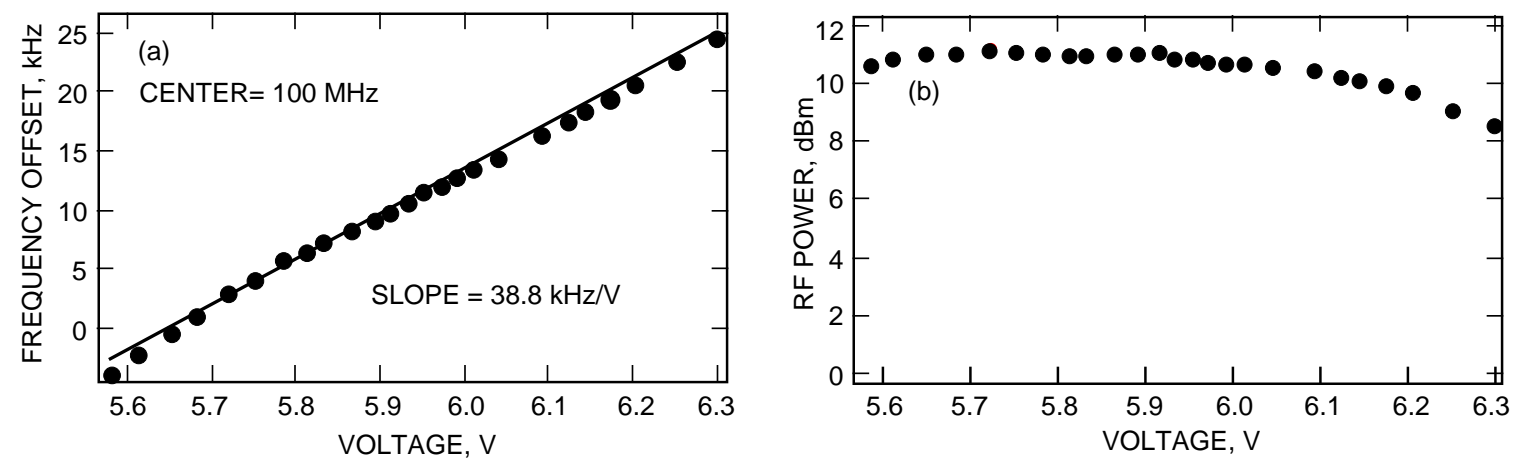

Fig. 4. Demonstration of the capability of tuning the photonic oscillator's frequency by controlling the bias voltage of the photonic modulator: (a) frequency versus bias voltage and (b) oscillation power versus bias voltage.

signal or an electrical signal, as shown in Fig. 1(b). Being able to injection lock an oscillator optically is important because it allows remote synchronization [14,15]. This function is critical for high-frequency RF systems that require many oscillators locked to a single master, as in a phased-array radar. Another advantage of optical injection locking is that the locking oscillator is electrically isolated from the locked oscillator, eliminating the need for impedance matching between the oscillators.

Figure 5(a) shows the experimental results of injection locking the photonic oscillator with a maser reference at $100 \mathrm{MHz}$ through the electrical injection port. Similar results are expected for optical injection since the optical injection signal will first be converted to an electrical signal by an internal photodetector in the loop before affecting the E/O modulator. As shown in Fig. 5, with an injection power of $-5 \mathrm{dBm}$, the phase noise of the photonic oscillator was almost identical to that of the injecting maser signal. Note that the output RF power of the photonic oscillator was $13 \mathrm{dBm}$, resulting in a gain of $18 \mathrm{~dB}$. As the injection power decreased, the phase noise of the photonic oscillator increased somewhat. However, the output RF power remained the same and, therefore, the gain was effectively increased. In the experiments, we were able to injection lock the photonic oscillator to a maser reference with an injection power as low as $-50 \mathrm{dBm}$. Figure 5 (b) shows the experimental result of the locking range as a function of injection power. As expected, the locking range is linearly proportional to the square root of the injection power, agreeing well with Adler's injection-locking theory [12].
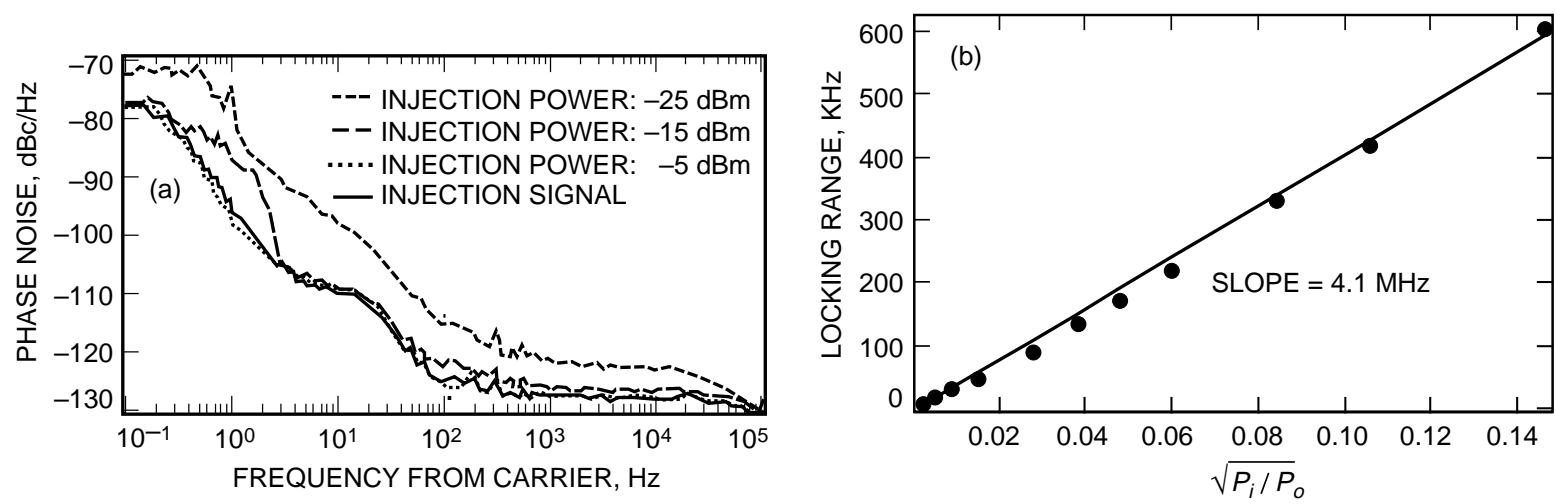

Fig. 5. Demonstration of injection locking the photonic oscillator: (a) phase noise measurement of the injectionlocked oscillator and (b) locking range as a function of the square root of the injection power.

\section{B. Self-Injection Locking}

Although injection locking is an effective means of synchronizing and stabilizing oscillators, it requires a low-noise and high-stability source to begin with. Making a high-frequency and high-stability source 
itself is a difficult task. By using a novel scheme called self-injection locking to stabilize the photonic oscillator, the photonic oscillator may be made as the frequency reference, as illustrated in Fig. 6(a). In this scheme, we derive a small portion of the output optical signal from the photonic oscillator and send it through a long fiber delay line. The output from the fiber delay line is then converted to electric signal and is fed back to the RF driving port of the E/O modulator. Note that the open-loop gain of this feedback loop should be kept much below unity so that no self-oscillation can be started. Basically, what we do here is to inject a delayed replica of the photonic oscillator's output back to the oscillator and force the oscillator to lock to its "past." This will prevent the oscillator from changing its frequency and phase and, hence, reduce the frequency and phase fluctuations. The frequency stability of the oscillator then is expected to be proportional to the length fluctuation $\Delta L / L$ of the fiber delay line.

Figure 6(b) presents the experimental results, showing the effectiveness of the self-injection technique in reducing the frequency noise of the photonic oscillator. The length of the delay line used in the experiment is $12 \mathrm{~km}$, and the feedback injection RF power is $-19.23 \mathrm{dBm}$. It is evident that self-injection locking greatly reduced the frequency fluctuations of the photonic oscillator. Further noise reduction is expected if we reduce the length fluctuation of the fiber delay line by placing it in a temperature-controlled environment and isolating it from acoustic vibrations. More experiments are under way to further reduce the noise of the self-injection locked photonic oscillator.

(a)
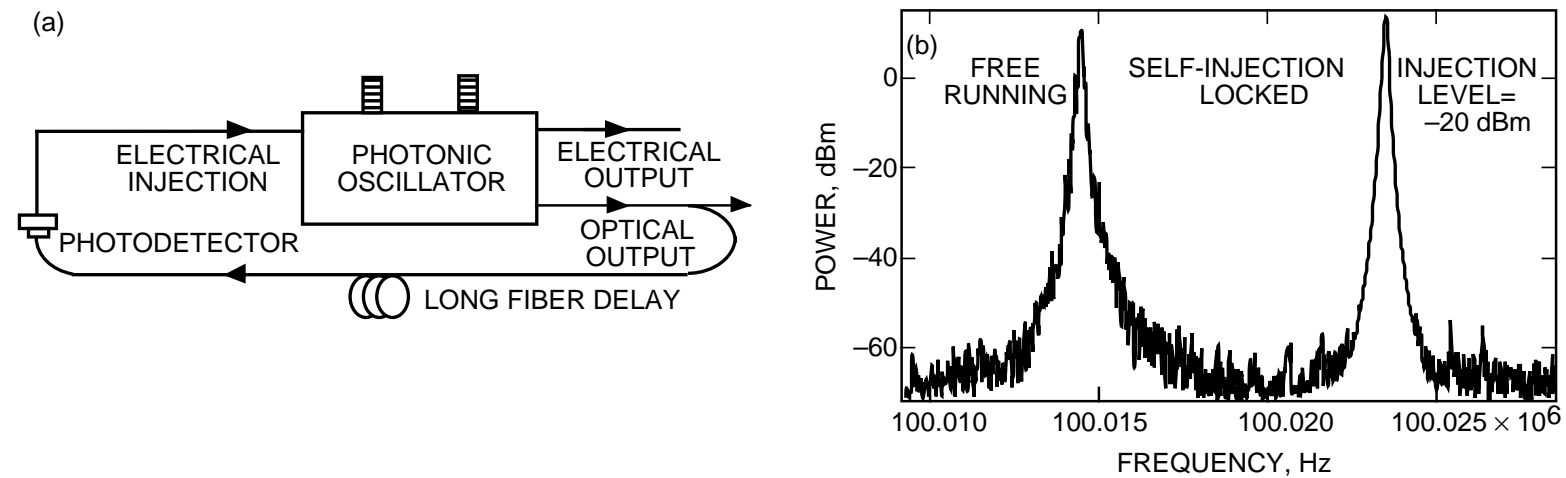

Fig. 6. Demonstration of self-injection locking: (a) the self-injection locking concept and (b) experimental result.

\section{Phase-Locked Loop}

Because the photonic oscillator is also a VCO, it can be synchronized to a reference source via a phase-locked loop [16], as shown in Fig. 7(a). Currently, experiments are under way for evaluating the performance of such a photonic phase-locked loop.

\section{Self-Phase Locking}

One unique property of the photonic oscillator is that it has an optical output. With this optical output, we can make a self-phase-locked loop to stabilize the photonic oscillator, as shown in Fig. 7(b). Similar to self-injection locking described earlier, a self-phase-locked loop forces the oscillator to be locked to its past and prevents it from fluctuating.

Using the delay-line discriminator technique to stabilize an oscillator is well known. To effectively stabilize an oscillator, a delay of many kilometers is needed and was, therefore, considered impractical before the emergence of the photonic technology. In our laboratory, Logan et al. [17] previously demonstrated using a fiber-optic delay line to stabilize a traditional VCO and obtained excellent results. However, in that setup, the fiber-optic delay line included a laser transmitter to convert the VCO's electrical output into optical signal and then transmit the optical signal through a few kilometers of fiber. Since the photonic oscillator automatically contains an optical output, it is ideal for using the fiber delay line technique 
(a)

BIAS

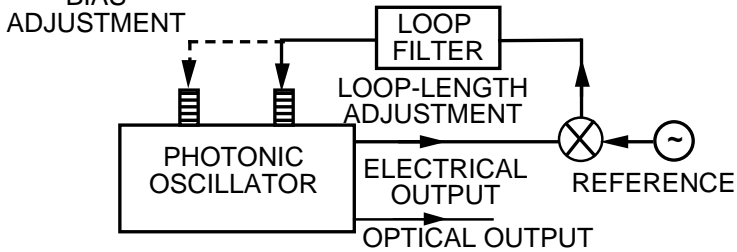

(b)

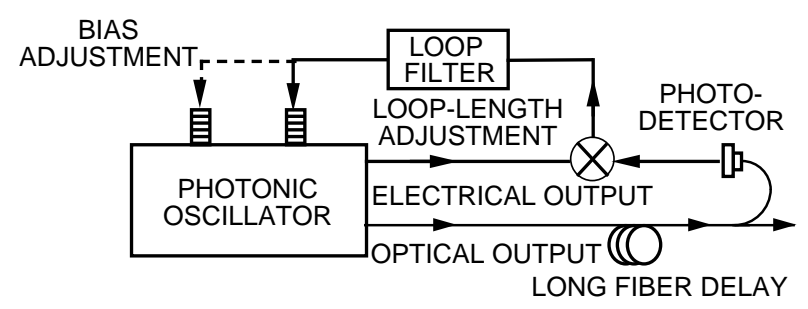

Fig. 7. Illustrations of phase locking the photonic oscillator using a phase-locked loop: (a) phase locking to a reference source and (b) phase locking to its past or self-phase locking.

to stabilize itself without the need of electrical-to-optical signal conversion. Consequently, the device is simple, low loss, and less expensive.

\section{Applications}

Voltage Controlled Oscillator: As mentioned earlier, the photonic oscillator is a special VCO with optical output. Therefore, it can perform all functions that a VCO is capable of for photonic RF systems. These functions include generating, tracking, cleaning, amplifying, and distributing RF carriers [16]. The photonic VCOs in a phase-locked loop configuration can also be used for clock recovery, carrier recovery, signal modulation and demodulation, and frequency synthesis.

Photonic Signal Mixing: The photonic oscillator can also be used for photonic signal up/down conversion, as shown in Fig. 8. For such an application, a stable optical RF LO, or a modulated optical signal at an RF frequency, is required. The photonic oscillator can accomplish just that, since one of its outputs gives the RF oscillation in optical domain.

Carrier Distribution: Because the photonic oscillator can be injection locked by a remote optical signal, it can be used for high-frequency RF carrier regeneration, amplification, and distribution, as shown in Fig. 9. Such a capability is important in large photonic RF systems.

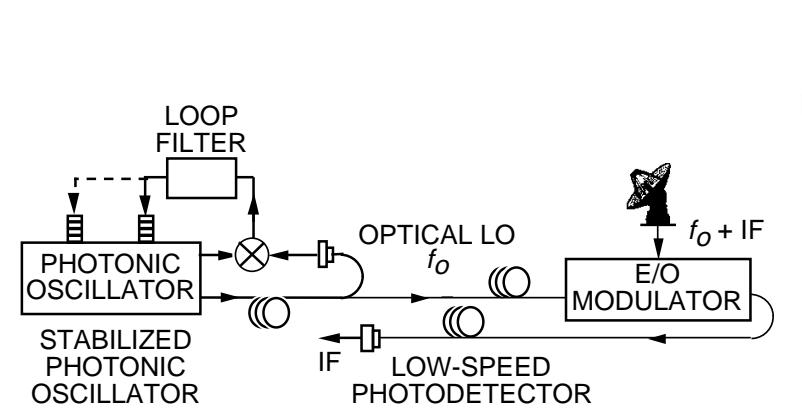

Fig. 8. Using the photonic oscillator for photonic downconversion.

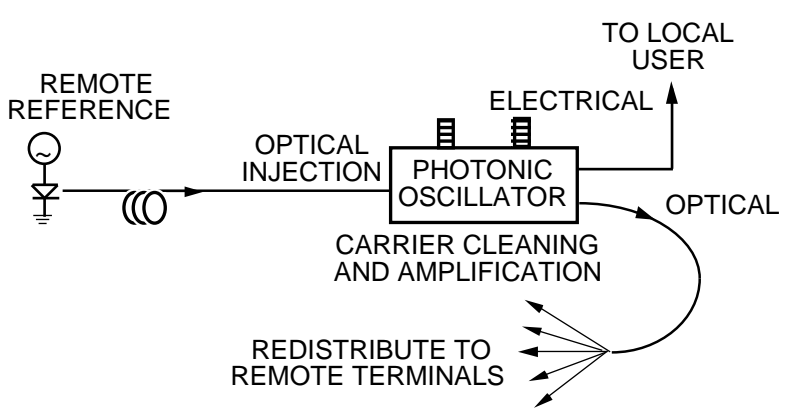

Fig. 9. Using the photonic oscillator for reference regeneration and distribution.

Frequency Multiplication: The injection-locking property of the photonic oscillator can also be used for high-gain frequency multiplication. In the first scheme, as shown in Fig. 10(a), the nonlinearity of the modulator is used and the photonic oscillator is injection locked to an external signal that is a subharmonic of the oscillator's operating frequency. This is the so-called subharmonic injection locking [18]. We have demonstrated phase locking the oscillator operating at $300 \mathrm{MHz}$ to a $100-\mathrm{MHz}$ reference of $4 \mathrm{dBm}$. The output of the oscillator is $15 \mathrm{dBm}$, resulting in a gain of $11 \mathrm{~dB}$ and a frequency multiplication factor of 3. 
(a)

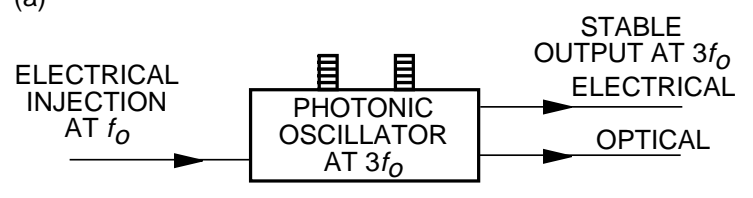

(b)

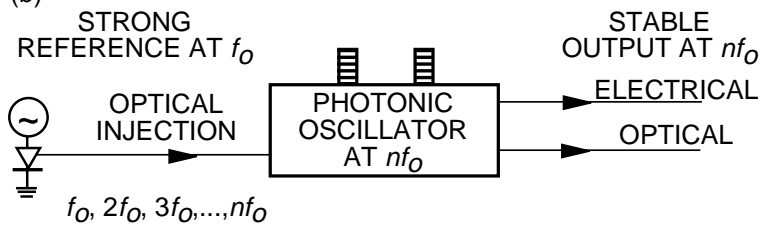

Fig. 10. Using the photonic oscillator for frequency multiplication: (a) using the photonic oscillator's nonlinearity and (b) using the laser diode's nonlinearity.

In the second scheme, the nonlinearity of a laser diode [14] is used, as shown in Fig. 10(b). If the laser is biased properly and is driven hard enough, its output will contain many harmonics of the driving signal. The photonic oscillator is tuned to operate at a nominal frequency close to the $n$th harmonic of the reference signal driving the laser diode. Upon the injection of the laser's output, the photonic oscillator will be locked to the $n$th harmonic. This scheme offers remote frequency multiplication capability [19] and may be useful for many photonic RF systems.

Comb-Frequency and Square-Wave Generation: The photonic oscillator can also be used to generate frequency combs and square waves, as shown in Fig. 11. For this application, the photonic oscillator is chosen to operate with multimodes. A sinusoidal signal with a frequency equal to the mode spacing or a multiple of mode spacings is injected into the oscillator. Just like laser mode locking, this injected signal will force all modes to oscillate in phase. Consequently, we obtain a comb of frequencies that are in phase. In the time domain, the output signal is a square wave.
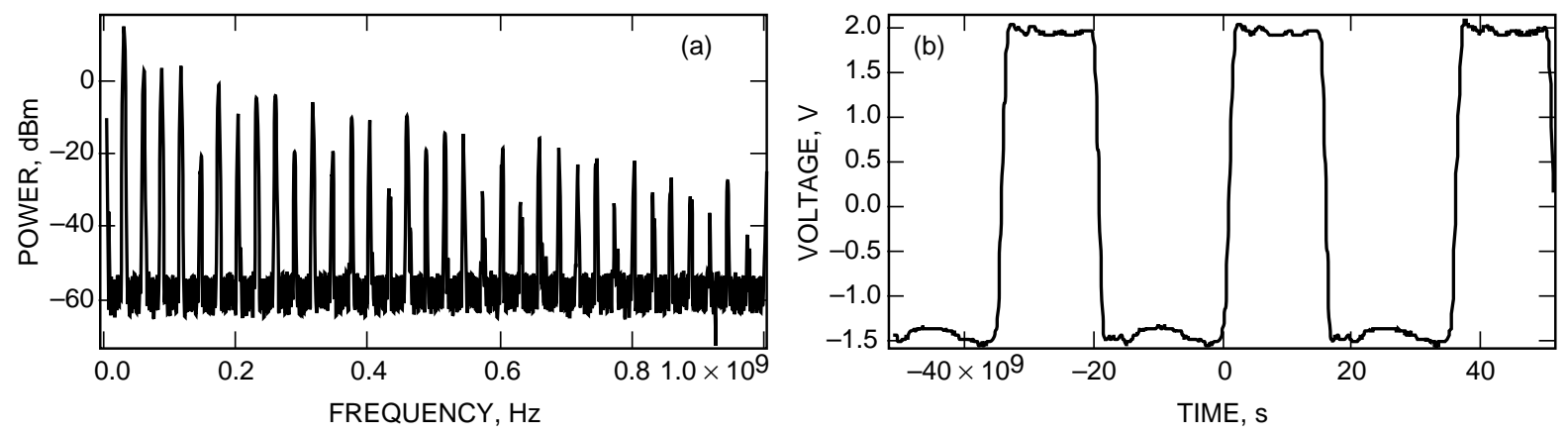

Fig. 11. Using the photonic oscillator for (a) comb-frequency generation and (b) square-wave generation.

Clock and Carrier Recovery: In high-speed fiber-optic communication systems, the ability to recover the clock from the incoming random data is essential [20,21]. The same injection-locking property of the photonic oscillator can also be used for clock and carrier recovery. The incoming data are injected into the photonic oscillator either optically or electrically. The free-running photonic oscillator is tuned to oscillate at a nominal frequency equal to the clock frequency of the incoming data. With the injection of the data, the photonic oscillator will be quickly phase locked to the clock frequency of the data stream while rejecting other frequency components (harmonics and subharmonics) associated with the data. Consequently, the output of the locked photonic oscillator is a continuous periodic wave synchronized with the incoming data, or simply the recovered clock. As can be seen, the device has both electrical and optical inputs and both electrical and optical outputs.

We have demonstrated clock recovery from both return-to-zero (RZ) and nonreturn-to-zero (NRZ) data at $100 \mathrm{Mb} / \mathrm{s}$ and have obtained excellent results [22]. Data rates up to $70 \mathrm{~Gb} / \mathrm{s}$ can also be recovered using the injection-locking technique with a photonic oscillator operating at $70 \mathrm{GHz}$. Note that the data rates at one-half of this value are impossible to achieve with the current electronic clock recovery techniques. 
Another important feature of the photonic oscillator technique is that the clock can be recovered directly from data just out of a fiber-optic transmission line, without the need of optical-to-electrical conversion. In addition, the recovered clock signal has both optical and electrical forms and is easy to interface with a fiber-optic communication system.

Similar to clock recovery, a carrier buried in noise can also be recovered by the photonic oscillator. To do so, we simply inject the spoiled carrier into a photonic oscillator that has a free-running frequency close to the carrier and an output power level $N(N>>1) \mathrm{dB}$ higher than the carrier. The injected carrier forces the photonic oscillator to be locked with the carrier and results in an equivalent carrier gain of $N \mathrm{~dB}$. Because the open-loop gain of the photonic oscillator is only $n \mathrm{~dB}(n \sim 1)$, the noise of the input is only amplified by $n \mathrm{~dB}$, and the signal-to-noise ratio of the carrier is then increase by $(N-n) \mathrm{dB}$. We have also demonstrated the recovery of carrier from noise and increased the carrier-to-noise ratio by $50 \mathrm{~dB}[22]$.

\section{Summary}

In summary, we have reported a novel photonic oscillator. This device is capable of generating stable signals at frequencies up to $70 \mathrm{GHz}$ and is a special VCO with both optical and electrical output. It can be used to make a phase-locked loop (PLL) and to perform all functions that a PLL is capable of for photonic systems. It can be synchronized to a reference source by means of optical injection locking, electrical injection locking, and PLL. It can also be self-phase locked and self-injection locked to generate high-stability photonic RF reference. Its applications include high-frequency reference regeneration and distribution, high-gain frequency multiplication, comb-frequency and square-wave generation, carrier recovery, and clock recovery. We anticipate that such photonic VCOs will be as important to photonic RF systems as electrical VCOs are to electrical RF systems.

\section{Acknowledgments}

We thank G. Lutes and M. Calhoun for many technical discussions and for their assistance.

\section{References}

[1] H. Ogawa, D. Polifko, and S. Banba, "Millimeter-Wave Fiber Optics Systems for Personal Radio Communication," IEEE Trans. Microwave Theory Techn., vol. 40, no. 12, pp. 2285-2293, 1992.

[2] P. Herczfeld and A. Daryoush, "Fiber Optic Feed Network for Large Aperture Phased Array Antennas," Microwave Journal, pp. 160-166, August 1987.

[3] X. S. Yao, G. Lutes, R. Logan, and L. Maleki, "Field Demonstration of X-Band Photonic Antenna Remoting in the Deep Space Network," The Telecommunications and Data Acquisition Progress Report 42-117, January-March 1994, Jet Propulsion Laboratory, Pasadena, California, pp. 29-34, May 15, 1994.

[4] G. K. Gopalakrishnan, W. K. Burns, and C. H. Bulmer, "Microwave-Optical Mixing in $\mathrm{LiNbO}_{3}$ Modulators," IEEE Trans. Microwave Theory Techn., vol. 41, no. 12, pp. 2383-2391, 1993.

[5] E. Toughlian and H. Zmuda, "A Photonic Variable RF Delay Line for Phased Array Antennas," J. of Lightwave Technology, vol. 8, pp. 1824-1828, 1990.

[6] X. S. Yao and L. Maleki, "A Novel 2-D Programmable Photonic Time-Delay Device for Millimeter-Wave Signal Processing Applications," IEEE Photonic Techn. Lett., vol. 6, no. 12, pp. 1463-1465, 1994. 
[7] D. Nortton, S. Johns, and R. Soref, "Tunable Wideband Microwave Transversal Filter Using High Dispersive Fiber Delay Lines," Proceedings of the 4th Biennial Department of Defense Fiber Optics and Photonics Conference, McLean, Virginia, pp. 297-301, 1994.

[8] B. Moslehi, K. Chau, and J. Goodman, "Fiber-Optic Signal Processors With Optical Gain and Reconfigurable Weights," Proceedings of the 4 th Biennial Department of Defense Fiber Optics and Photonics Conference, McLean, Virginia, pp. 303-309, 1994.

[9] Introduction to Diode-Pumped Solid-State Lasers, Lightwave Electronics Corp., Technical Information No. 1, Mountain View, California, 1993.

[10] X. S. Yao and L. Maleki, "High Frequency Optical Subcarrier Generator," Electron. Lett., vol. 30, no. 18, pp. 1525-1526, 1994.

[11] X. S. Yao and L. Maleki, "Influence of an Externally Modulated Photonic Link on a Microwave Communications System," The Telecommunications and Data Acquisition Progress Report 42-117, January-March 1994, Jet Propulsion Laboratory, Pasadena, California, pp. 16-28, May 15, 1994.

[12] R. Adler, "A Study of Locking Phenomena in Oscillators," Proc. IRE, vol. 34, pp. 351-357, 1946.

[13] K. Kurokawa, "Injection Locking of Microwave Solid-State Oscillators," Proceedings of the IEEE, vol. 61, no. 10, pp. 1386-1410, 1973.

[14] A. Daryoush, "Optical Synchronization of Millimeter-Wave Oscillators for Distributed Architectures," IEEE Trans. Microwave Theory Techn., vol. 38, no. 5, pp. 467-476, 1990.

[15] L. Goldberg, C. Rauscher, J. F. Weller, and H. F. Taylor, "Optical Injection Locking of X-Band FET Oscillator Using Coherent Mixing of GaAs Lasers," Electron. Lett., vol. 19, no. 20, pp. 848-849, 1983.

[16] D. Wolaver, Phase-Locked Loop Circuit Design, Englewood Cliffs, New Jersey: Prentice Hall, 1991.

[17] R. Logan, L. Maleki, and M. Shadaram, "Stabilization of Oscillator Phase Using a Fiber Optic Delay Line," Proceedings of the 45th Annual Symposium on Frequency Control, IEEE Ultrasonic Ferroelectric and Frequency Control Society, Los Angeles, California, May 29-31, 1991.

[18] X. Zhang, X. Zhou, and A. Daryoush, "A Theoretical Study of the Noise Behavior of Subharmonically Injection Locked Local Oscillators," IEEE Trans. Microwave Theory Techn., vol. 40, no. 5, pp. 895-902, 1992.

[19] A. Daryoush, P. Herczfeld, Z. Turski, and P. Kahi, "Comparison of Indirect Optical Injection Locking Techniques of Multiple X-Band Oscillators," IEEE Trans. Microwave Theory Techn., vol. MTT-34, no. 12, pp. 1363-1369, 1986.

[20] P. E. Barnsley, H. J. Wickes, G. E. Wickens, and D. M. Spirit, "All-Optical Clock Recovery From $5 \mathrm{~Gb} / \mathrm{s}$ Data Using a Self-Pulsation $1.56 \mathrm{~mm}$ Laser Diode," IEEE Photonic Techn. Lett., vol. 3, no. 10, pp. 942-945, 1991.

[21] D. M. Patrick and R. J. Manning, "20 Gbit/s All-Optical Clock Recovery Using Semiconductor Nonlinearity," Electron. Lett., vol. 30, no. 2, pp. 151-152, 1994.

[22] X. S. Yao and G. Lutes, "A High-Speed Photonic Clock and Carrier Regenerator," The Telecommunications and Data Acquisition Progress Report 42-121, January-March 1995, Jet Propulsion Laboratory, Pasadena, California, pp. 202210, May 15, 1995. 